\title{
Gestational Hypertension
}

National Cancer Institute

\section{Source}

National Cancer Institute. Gestational Hypertension. NCI Thesaurus. Code C4371.

A blood pressure elevation after 20 weeks of gestation in the absence of either proteinuria or systemic findings like thrombocytopenia, impaired liver function, progressive renal insufficiency, pulmonary edema or the new-onset of cerebral or visual disturbances. 